Mesoscopic Physics in Complex Media, 03008 (2010)

DOI:10.1051/iesc/2010mpcm03008

(C) Owned by the authors, published by EDP Sciences, 2010

\title{
PROBING DECOHERENCE THROUGH FANO RESONANCES
}

\author{
A. Bärnthaler ${ }^{1}$, S. Gehler ${ }^{2}$, S. Rotter ${ }^{1, *}$, F. Libisch ${ }^{1}$, U. Kuhl ${ }^{2}$, H.-J. Stöckmann ${ }^{2}$, J. Burgdörfer ${ }^{1}$ \\ ${ }^{1}$ Institute for Theoretical Physics, Vienna University of Technology, A-1040Vienna, Austria, *stefan.rotter@tuwien.ac.at \\ ${ }^{2}$ Fachbereich Physik, Philipps-Universität Marburg, Renthof 5, D-35032 Marburg, Germany
}

This is an Open Access article distributed under the terms of the Creative Commons Attribution-Noncommercial License 3.0, which permits unrestricted use, distribution, and reproduction in any noncommercial medium, provided the original work is properly cited. 


\section{Probing DeCOHEREnCE THRough FAno RESOnANCES}

A. Bärnthaler ${ }^{1}$, S. Gehler ${ }^{2}$, S. Rotter ${ }^{1, *}$, F. Libisch ${ }^{1}$, U. Kuhl ${ }^{2}$, H.-J. Stöckmann ${ }^{2}$, J. Burgdörfer

${ }^{1}$ Institute for Theoretical Physics, Vienna University of Technology, A-1040 Vienna, Austria, *stefan.rotter@tuwien.ac.at ${ }^{2}$ Fachbereich Physik, Philipps-Universität Marburg, Renthof 5, D-35032 Marburg, Germany

\section{Tuning Fano Resonances}

Fano Resonances

Fano resonances arise in transmission if there is

- a single resonant term

- an additional approximately constant background $t_{d}$

Examples: neutron scattering, dissipation spectra, quantum dots

Transmission amplitude:

$$
t(k)=z_{r} \frac{\Gamma / 2}{k-k_{\mathrm{res}}+i \Gamma / 2}+t_{d}
$$

$$
|t(k)|^{2}=\left|t_{d}\right|^{2} \frac{|\epsilon+q|^{2}}{\epsilon^{2}+1}, \text { with } q=i+\frac{z_{r}}{t_{d}}
$$

$\bullet \quad \epsilon=\left(k-k_{\mathrm{res}}\right) /(\Gamma / 2):$ rescaled wavenumber

- $q$ : Fano parameter describes the asymmetry of the resonance

- $q \rightarrow 0 \Rightarrow$ window or "anti-resonance"

$\bullet \rightarrow \pm \infty \Rightarrow$ Breit-Wigner resonance

\section{Microwave Experiments - Quantum Mechanics}

From Maxwell's equation for microwave resonators with parallel bottom and top plate we obtain the two-dimensional Helmholtz-equatio for the $z$-component of the electrical field $E_{z}(x, y)$ :

$$
-\left(\frac{\partial^{2}}{\partial x^{2}}+\frac{\partial^{2}}{\partial y^{2}}\right) E_{z}(x, y)=k^{2} E_{z}(x, y),
$$

There exists a full correspondence between Eq. (1) with the twodimensional Schrödinger-equation

$$
-\frac{\hbar^{2}}{2 m}\left(\frac{\partial^{2}}{\partial x^{2}}+\frac{\partial^{2}}{\partial y^{2}}\right) \psi(x, y)=E \psi(x, y)
$$

if we identify

$$
\psi \hat{=} E_{z}, \quad E \hat{=} k^{2}, \quad \frac{\hbar^{2}}{2 m} \hat{=} 1
$$

$1.07 w / d=37 \%$
Experimental set-up

- Rectangular copper cavity with

width $D=39 \mathrm{~mm}$ and length $L=176 \mathrm{~mm}$

- Excite via channels of width $d=15.8 \mathrm{~mm}$

- Varying shutter opening $w$ changes coupling

$(1.8 \mathrm{~mm} \leq w \leq 15.8 \mathrm{~mm})$

- Mode with $n=2$ cannot be excited due to symmetry

- Coupling to modes with $n=1$ and $n=3$ is different

- Measure complex transmission amplitude $t$

with vector network analyzer

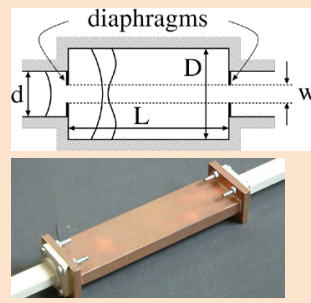

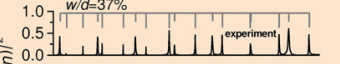

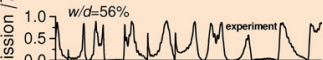

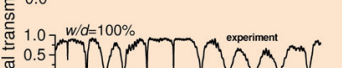

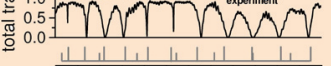

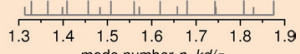

Transmission Spectra

- Transmission $|t(n)|^{2}$ for different shutter opening

- Shutter opening $d=5.8 \mathrm{~mm}$ : Breit-Wigner resonances

- Shutter opening $d=7.8 \mathrm{~mm}$ : asymmetric resonances

- Shutter opening $d=15.8 \mathrm{~mm}$ : window resonances

- Positions of all eigenstates in the closed cavity

are indicated by the gray tick marks

- Long gray ticks marks for $n=3$ and short for $n=1$ eigenstates

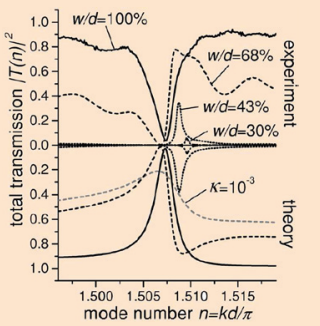

Individual Fano Resonance

- Effects of dissipation have to be taken into account $\left(\kappa=10^{-4}\right)$

- Shape is sensitive to the dissipation parameter (see $\kappa=10^{-3}$ curve)

- Fano resonances can be tuned

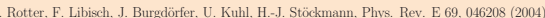

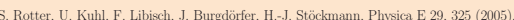

Probing decoherence through Fano resonances [Phys. Rev. Lett. 105, 056801 (2010)]

Model for Dissipation

Dissipation is modelled by many, weakly attached channels, where

the flux leaves the cavity.

Effective description

$k \rightarrow k+i \kappa$

$q(\chi)=i+\chi \frac{z_{r}}{t_{d}}=i+\chi\left(q_{0}-i\right)$

$\chi=\Gamma_{0} /\left(\Gamma_{0}+2 \kappa\right)$ : measure for th dissipation strength

$q_{0}$ : Fano parameter for the system.

without dissipatio

$q(\chi)$ : straight line from $q_{0}$ to

Model for Dephasing

Dephasing is modelled by attached phase breaking channel where the flux into the channel is re-injected with an arbitrary phase.

Also dephasing leads to a complex Fano parameter, but with a circular dependence on the dephasing strength $\chi$

$q(\chi)$ : circular dependence

$\chi=\Gamma_{0} / \Gamma$ : measure for the dephasing strength

A. A. Clerk, X. Waintal, and P. W. Brouwe Phys. Rev. Lett. 86, 4636 (2001).]

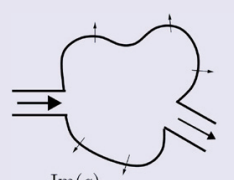

Experimental and Numerical Results

- Three different cavities: copper, brass, and steel

(same size as cavity shown above)

- Two temperatures: room temperature and liquid nitrogen

- Shutter opening: $d=7.8 \mathrm{~mm}$
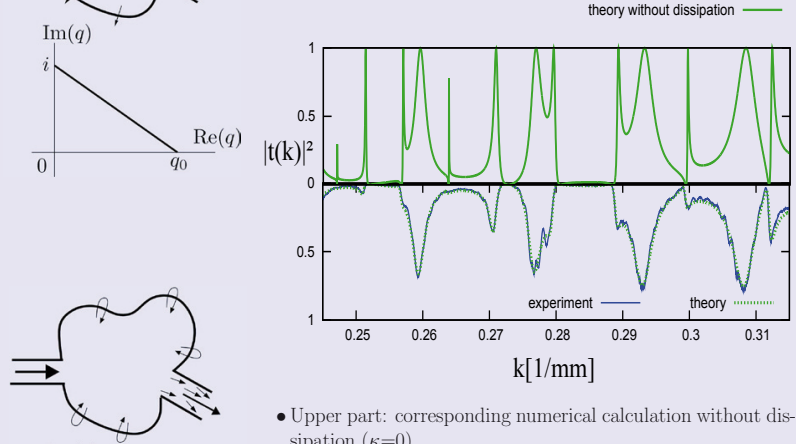

$\mathrm{k}[1 / \mathrm{mm}]$ $\operatorname{Im}(q)$

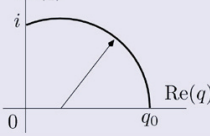

- Upper part: corresponding numerical calculation without dissipation $(\kappa=0)$

- Lower part: measured transmission for steel cavity and numerical calculation with dissipation ( $\kappa$ is fitted)

- Numerical data coincides very well with experimental data

- Well controlled dissipation in experiment allows us to study the influence on Fano resonances explicitly
Scaling for Dissipation

- Resonances in microwave transmission fitted with Fano lineshape - Fano parameter extracted for resonances with different materia parameters and temperatures

- Parameters resized to $q_{0}=1$

- Symbols for the same resonances are connected (empty/filled symbols stand for nitrogen/room temperature)

- Linear scaling behavior is successfully reproduced

Scaling for Dephasing

- Numerical calculation with uniform dephasing and experimental data on temperature dependence of transport through quantum dots [PRB 64, 155311 (2001)]

- Parameters resized to unit circle

- Circular scaling behavior is suc-

cessfully reproduced

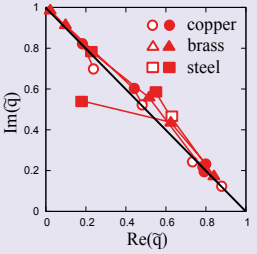

Parametric dependence of the Fano asymmetry parameter $q(\chi)$ can distinguish between different decoherence mechanisms 